\title{
Promoting Metro Operation Safety by Exploring Metro Operation Accident Network
}

\author{
Qi SUO \\ School of Economics and Management, Qingdao University of Science and Technology, Qingdao \\ 266061, China \\ E-mail: suoqi1980@163.com \\ Liyuan WANG \\ School of Economics and Management, Qingdao University of Science and Technology, Qingdao \\ 266061, China \\ E-mail: 1720270573@qq.com \\ Tianzi YAO \\ School of Economics and Management, Qingdao University of Science and Technology, Qingdao \\ 266061, China \\ E-mail: 850414476@qq.com \\ Zihao WANG \\ School of Economics and Management, Qingdao University of Science and Technology, Qingdao \\ 266061, China \\ E-mail: 923784925@qq.com
}

\begin{abstract}
Understanding the causation of accidents is essential to promote metro operation safety. In terms of 243 reported metro operation accident cases in China, a directed weighted network was constructed based on complex network theory, where nodes and directed edges denotes factors and event chains respectively. To reveal the key causal factors, the topological characteristics of metro operation accident network (MOAN) were analyzed from both global and local views. The results show that facility-type factors are more closely related to the occurrence of the accidents from the perspectives of average path length and cascading effects. Accident types like train delay and train suspension are the great risk recipients. Key causal factors with large out-degree, out-strength, betweenness centrality and cluster coefficient, such as communication and signal failure, vehicle failure and piling into the train should be noticed. The research framework proposed in the paper is not only applicable to China's metro operation system, but also appropriate for other transportation system safety studies.
\end{abstract}

Keywords operation accident analysis; directed weighted network; metro operation accident network (MOAN); complex network

Received January 3, 2021, accepted February 24, 2021

Supported by the National Natural Science Foundation of China (NSFC) (71801139), Qingdao Social Science Planning Project (QDSKL1801157), and Key Research and Development Plan (Soft Science Project) of Shandong Province (2019RKB01118) 


\section{Introduction}

Metro is a complex system composed of "man-machine-electricity", whose subsystems are closely related to each other. Thus metro operation accidents invariably do not result from a single factor, but from a combination of person, facility, environment and management factors. In considering the complexity of the causation, systemic accident models should be introduced. There were three noted methods in such models, namely Accimap ${ }^{[1]}$, human factors analysis and classification system (HFACS) ${ }^{[2]}$ and systems theoretic accident modelling and processes model (STAMP) ${ }^{[3]}$. Many efforts have been made to use these three methods to reveal how accidents occur ${ }^{[4-6]}$. However, systemic accident models mostly adopt qualitative analysis and the results may be subject to the subjective emotion of the respondents.

To make up for the above shortcoming, complex network method was utilized to make research more objective and credible by analyzing topological structure of the network. In recent years, the complex network method has been widely used in different fields, such as environment $^{[7]}$, economy ${ }^{[8,9]}$, mechanical engineering ${ }^{[10,11]}$, safety ${ }^{[12-14]}$, etc. In the field of metro operation safety, many studies focus on metro network analysis using complex network. $\mathrm{Ma}$, et al. ${ }^{[15]}$ revealed key causal factors from the overall perspective based on "7.23 China Yongwen railway accident". Li, et al. ${ }^{[16]}$ collected 134 accidents occurred in China and used seven parameters to further explore the topological structure of the directed network. Liu, et al. ${ }^{[17]}$ proposed a new network theory-based approach and made comparison of the topological analysis results with the conventional method. Lam and Tai ${ }^{[18]}$ analyzed 125 reported railway accidents in Japan from local, global and contextual views.

All the above studies have played an important role in finding causal factors and improving safety, however, the weight of the relationships has not been take into consideration. It's well known that network graphs based on the complex network theory can be divided into four categories, including undirected unweighted network, undirected weighted network, directed unweighted network and directed weighted network. Obviously, the fourth network conveys more information than others. Zhou, et al. ${ }^{[19]}$ established a directed weighted accident causation network and found the network has properties of small-world and scale-free. Hou, et al. ${ }^{[20]}$ reported important factors with higher degree, strength and betweenness centrality should be treated seriously. Du, et al. ${ }^{[21]}$ took Shenzhen as a case study to identify important nodes using a novel method.

To sum up, fewer studies paid attention to construct a directed weighted metro operation safety network based on historical accidents occurred in all over the China. However, China occupies an important position in the world's metro development progress. Taking it as a case study has an extremely representative and analytical value. Therefore, based on metro operation accidents occurred in China from 1972-2019, the paper presents a directed weighted metro operation accident network (MOAN), taking factors and event chains as nodes and edges respectively. Then the topological structures of MOAN are further explored using complex network theory from global and local views. Finally, in the terms of the statistics and analysis of MOAN, some countermeasures and further work are presented. 


\section{Materials and Methods}

\subsection{Collect and Filter Accidents}

China operated its first metro line on January 15, 1971 and a total of 44 cities have already operated metro up to December 31, 2019. The paper collected past accident cases using keywords such as "metro/subway/rail transit/underground", "safety" and "accident/incident" to search in official media and research literature. In total, 442 metro operation accidents between July 19, 1972 and December 31, 2019 are obtained. It should be notice the accident reports should include time, process, cause and the result of the accident, so cases that either only has single casual factor or brief descriptions are excluded from the database. Finally, the metro operation accident database contains 243 classic accident cases.

\subsection{Identify Causal Factors}

Accurate classification of causal factors will help to take effective measures to improve metro operation safety. Based on the metro operation risk lists of the "Metro Operation Safety Evaluation Standards" (GB/T 50438-2007) and the description of accidents, causal factors are categorized into four types, including person-type factors $(\mathrm{P})$, facility-type factors $(\mathrm{F})$, environmental-type factors (E) and management-type factors (M). In detail, 40 causal factors of metro operation accidents are extracted and shown in Table 1.

Table 1 Causal factors of metro operation accidents

\begin{tabular}{|c|c|c|}
\hline Level one & Level two & Level three \\
\hline \multirow[t]{3}{*}{ Person $(\mathrm{P})$} & Passenger & $\begin{array}{l}\text { P1 faint; P2 carrying dangerous articles; P } 3 \text { using the } \\
\text { emergency brake without authorization; P4 piling into } \\
\text { the train; P5 opening the door forcedly; P6 climbing } \\
\text { over the screen door; P7 fabricating false information; } \\
\text { P8 falling into platform by accident; P9 being pinched } \\
\text { in train door; P10 graffiti; P11 leaving things behind; } \\
\text { P12 conflict and assault; P13 tumble; P14 illegally } \\
\text { entering into the track area; }\end{array}$ \\
\hline & Driver & $\begin{array}{l}\text { P15 driver's wrong operation; P16 lack of safety risk } \\
\text { awareness; P17 lack of work experience; P18 fatigue; }\end{array}$ \\
\hline & $\begin{array}{l}\text { Management } \\
\text { personnel }\end{array}$ & $\begin{array}{l}\text { P19 management personnels wrong operation; P20 } \\
\text { inadequate ability to deal with emergencies; P21 } \\
\text { rule-breaking operation; }\end{array}$ \\
\hline \multirow{3}{*}{ Facility (F) } & The train & F1 screen door failure; F2 vehicle failure; \\
\hline & The track & $\begin{array}{l}\text { F3 catenary system failure; F4 track damage(including } \\
\text { turnout); F5 foreign bodies in the track; F6 water in } \\
\text { the tunnel; }\end{array}$ \\
\hline & Supporting facilities & $\begin{array}{l}\text { F7 communication and signal failure; F8 power supply } \\
\text { system failure; F9 propulsion system failure; F10 } \\
\text { escalator failure; }\end{array}$ \\
\hline \multirow{2}{*}{ Environment (E) } & Natural environment & E1 natural disasters; E2 severe weather; \\
\hline & Social environment & E3 terrorism or riots; E4 smoke; E5 gas leakage; \\
\hline \multirow[t]{2}{*}{ Management (M) } & $\begin{array}{l}\text { Management } \\
\text { organization }\end{array}$ & $\begin{array}{l}\text { M1 poor routine safety maintenance and inspection; } \\
\text { M2 inadequate education and training; M3 inadequate } \\
\text { safety supervision and management; }\end{array}$ \\
\hline & Emergency handling & M4 imperfect emergency rescue system \\
\hline
\end{tabular}


Similarly, the metro operation accidents are divided into 6 categories, as shown in Table 2. It is worth mentioning that several accident outcomes may simultaneously emerge in one single accident, so the total number of accidents caused by 243 cases is 265 . The statistics of metro operation accidents is presented in Figure 1. It shows that train delay accidents rank first, accounting for $62.40 \%$. It is followed by train suspension (21.60\%), and the other accident types $(16 \%$ in total).

Table 2 Metro operation accident types

A1 train suspension; A2 train delay; A3 fire;
Accident type (A) $\quad$ A4 collision (with human and train); A5 derailment;
A6 congestion and stampede

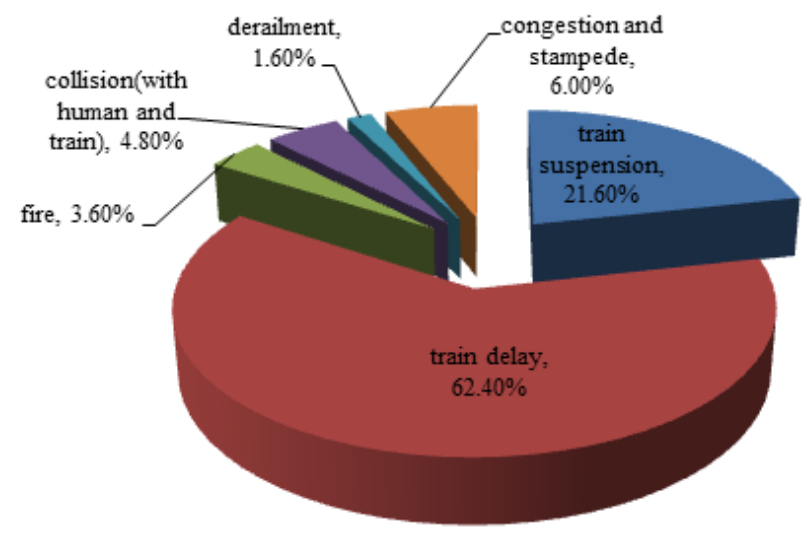

Figure 1 Statistics of various types of metro operation accidents

\subsection{Network Modeling}

Event chains can delineate the procedure of accidents in a simple and intuitive way. Obviously, most event chains are short, and two event chains can be formulated from one case. Using two accident cases as an example, the outline of the constructing event chain process is given in Table 3. In case 46, due to poor routine safety maintenance and inspection, the vehicle malfunctioned, directly causing a 46-minute delay. According to the description, the first event chain can be abstracted as "poor routine safety maintenance and inspection $\rightarrow$ vehicle failure $\rightarrow$ train delay". At the same time, without inadequate education and training, the driver failed to take effective and correct emergency measures in time after the vehicle broke down, hence the driver's wrong operation indirectly led to the accident. Therefore, the second event chain can be abstracted as "inadequate education and training $\rightarrow$ driver's wrong operation $\rightarrow$ train delay". 
Table 3 Two examples of identifying the event chains process

\begin{tabular}{|c|c|c|c|c|}
\hline $\begin{array}{c}\text { Case } \\
\text { no. }\end{array}$ & City & Time & Description of incidents & Event chains \\
\hline 46 & Beijing & $\begin{array}{c}15 \\
\text { March } \\
2013\end{array}$ & $\begin{array}{l}\text { At } 8 \text { AM, a train on the Muxidi platform broke down } \\
\text { when it departed, causing a 46-minute delay directly. } \\
\text { And the vehicle fault also reflected the staff's poor } \\
\text { routine safety maintenance and inspection. At the } \\
\text { same time, due to the lack of comprehensive and } \\
\text { effective education and training, the driver did not } \\
\text { press the reset button on the driver's platform in } \\
\text { accordance with the "provisional emergency treatment } \\
\text { measures" when facing with the accident, which } \\
\text { resulted in vehicle failure existing, the impact of the } \\
\text { fault expanding and the situation of train delay } \\
\text { happening ultimately. }\end{array}$ & $\begin{array}{l}\mathrm{M} 1 \rightarrow \mathrm{F} 2 \rightarrow \mathrm{A} 2 \\
\mathrm{M} 2 \rightarrow \mathrm{P} 15 \rightarrow \mathrm{A} 2\end{array}$ \\
\hline 88 & Shanghai & $\begin{array}{c}26 \\
\text { July } \\
2019\end{array}$ & $\begin{array}{l}\text { At around 7:45 PM, a passenger knocked on the door } \\
\text { of a train and used the emergency brake without } \\
\text { authorization on line } 4 \text { between the Shanghai railway } \\
\text { station and Zhongtan road, which caused the vehicle } \\
\text { to break down instantly. The train returned to normal } \\
\text { after the delay of several seconds. }\end{array}$ & $\mathrm{P} 3 \rightarrow \mathrm{F} 2 \rightarrow \mathrm{A} 2$ \\
\hline
\end{tabular}

Obviously, metro operation accident network (MOAN) with nodes denoted by factors and the directed edges denoted by event chains. And the weight of an edge represents the total number of occurrences. Thereby a directed weighted network is presented in Figure 2. The MOAN composed of 46 nodes and 265 event chains, including 40 causal factors and 6 accident types. Also, 265 events chains mean 327 relationships between pairs of nodes.

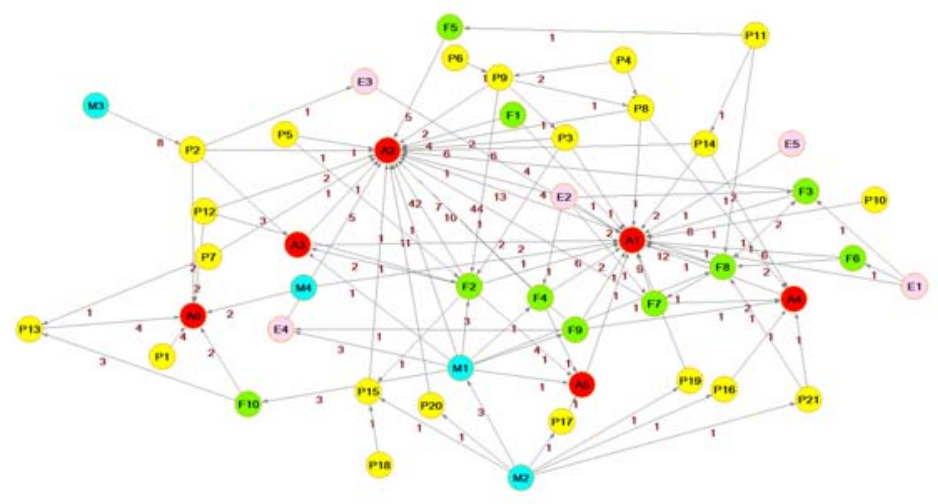

Figure 2 Metro operation accident network (MOAN) 
In Figure 2, Yellow color: Person-type factors; green color: Facility-type factors; pink color: Environment-type factors; blue color: Management-type factors; red color: Accident types.

\section{Results Analysis}

\subsection{Global View Analysis}

\subsubsection{Network Diameter}

Network diameter is defined as the longest path distance in the network, as shown in Equation (1).

$$
\text { Diameter }={ }_{i, j}^{\max } d_{i j}
$$

where $i$ represents a causal factor, $j$ represents a causal factor/accident type.

The diameter of MOAN is 4 . Take path that starts with "E1 (natural disaster)" and ends with "A4 (collision (with human and train))" as an example, the specific path includes the following two: $\mathrm{E} 1 \rightarrow \mathrm{F} 3 \rightarrow \mathrm{F} 8 \rightarrow \mathrm{F} 7 \rightarrow \mathrm{A} 4$ and $\mathrm{E} 1 \rightarrow \mathrm{F} 6 \rightarrow \mathrm{F} 8 \rightarrow \mathrm{F} 7 \rightarrow \mathrm{A} 4$. That is to say, once natural disasters occur, they may lead to the catenary system failure or the problem of water in the tunnel, thus lead to the power supply system failure and communication and signal failure. At last, the accident of collision (with human and train) happens. This fault propagation path reflects the process of risk diffusion.

\subsubsection{Network Density}

The network density refers to the degree of affinity among nodes. The higher the network density is, the closer the connection between nodes is. The calculation is given by Equation (2).

$$
D=\frac{2 M}{N(N-1)},
$$

where $M$ is the number of actual relationships and $\frac{N(N-1)}{2}$ is the number of nodes in the network.

The density of MOAN is 0.0502 , indicating that it is a sparse network and there are fewer direct connections among nodes. From the perspective of operation safety, forming a sparse network is a valuable method.

\subsubsection{Average Path Length}

The average path length is used to measure the accessibility between each node and reflects the efficiency of fault propagation. The shorter the average path length is, the higher the diffusion efficiency is. The calculation is given by Equation (3).

$$
L=\frac{1}{N(N-1)} \sum_{i, j \in N(i \neq j)} d_{i j},
$$

where $d_{i j}$ presents the shortest distance from node $i$ to node $j$.

The average path length of MOAN is 1.552. This demonstrates that a causal factor can cascade to another only in less than 2 steps on average. In other words, once there is a fault, it is easy to cause other faults quickly. 
Table 4 shows how different types of causal factors cause accidents. From Table 4, facilitytype factors are more likely to cause accidents only in 1.65 steps, followed by the environmenttype (1.70 steps). Besides, facility-type factors easily result in "A3 (fire)", "A5 (derailment)", "A6 (congestion and stampede)" only in one step and lead to "A2 (train delay)" only in 1.56 steps. Among four accident types, "A6 (congestion and stampede)" is the most likely accident only in 1.5 steps, followed by "A2 (train delay)".

Table 4 Average path lengths for different types of causal factors to cause accidents

\begin{tabular}{cccccccc}
\hline Factors & A1 & A2 & A3 & A4 & A5 & A6 & A \\
\hline Type P & 1.87 & 1.53 & 2.14 & 1.75 & 2.14 & 1.20 & 1.77 \\
Type F & 1.88 & 1.56 & 1.00 & 2.00 & 1.00 & 1.00 & 1.65 \\
Type E & 1.25 & 1.33 & - & 3.00 & 2.00 & - & 1.70 \\
Type M & 2.00 & 1.67 & 1.67 & 2.50 & 1.50 & 2.00 & 1.89 \\
All types & 1.81 & 1.53 & 1.91 & 2.06 & 1.83 & 1.50 & 1.76 \\
\hline
\end{tabular}

Figure 3 shows how similar types of causal factors cause accidents. From Figure 3(a), it can be found that the person-type factors "P1 (faint)", "P8 (falling into platform by accident)", "P10 (passengers graffiti)", "P13 (tumble)", "P14 (illegally entering into the track area)", "P15 (illegally entering into the track area)", "P19 (management personnel's wrong operation)" and "P20 (inadequate ability to deal with emergencies)" can easily result in accidents, given the value of one step. In Figure 3(b), some important facility-type factors can be identified, such as "F1 (screen door failure)", "F4 (track damage (including turnout))", "F5 (foreign bodies in the track)" and "F10 (escalator failure)". Similarly, in Figure 3(c) and (d), some key environmenttype and management-type factors can be determined, such as "E3 (terrorism or riots)", "E4 (smoke)", "E5 (gas leakage)" and "M4 (imperfect emergency rescue system)". The above factors have fewer steps potentially causing accidents than other causal factors from similar types, so we should pay more attention to them.

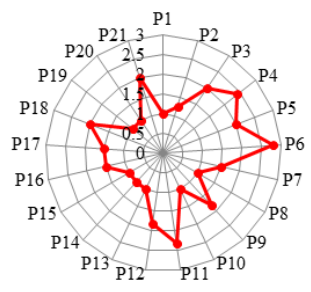

(a) person-type

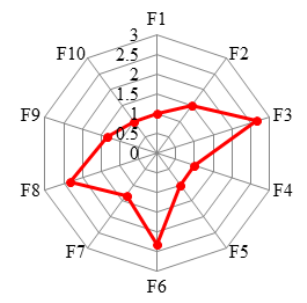

(b) facility-type

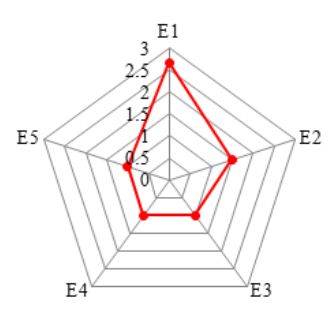

(c) environment-type

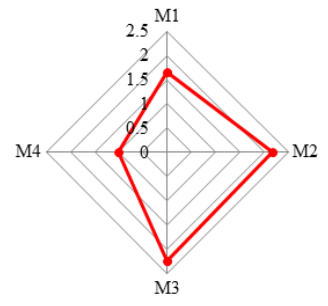

(d) management-type

Figure 3 Average path length for similar type of causal factors to cause accidents 


\subsubsection{Cascading Effects}

In Figure 4, P, F, M, E and A represent person, facility, management, environment and accident type respectively. The thickness of the directed edge represents the degree of tightness. As presented in Figure 4, the number of accidents caused by facility-type, person-type and environment-type factors is 178,54 , and 17 , respectively. It is approximately $67.17 \%$ of metro operation accidents related to facility failure directly. That is to say, facility-type factors are found to be comparatively significant causal factors. In Figure 5, facility-type factors are mainly related to "A2(train delay)", which is consistent with the fact that facility-type factors cause "A2(train delay)" only in 1.56 steps.

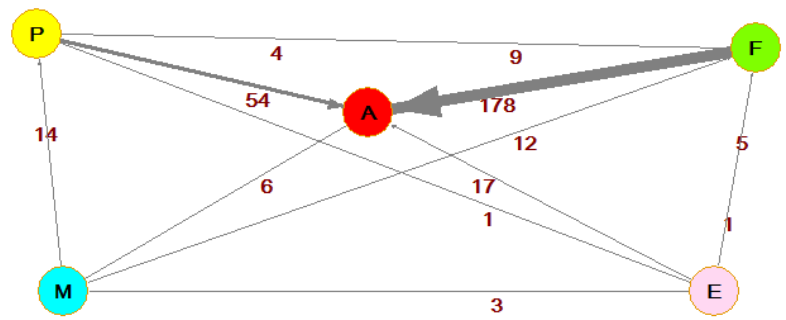

Figure 4 Cascading effects between causal factors and accident types

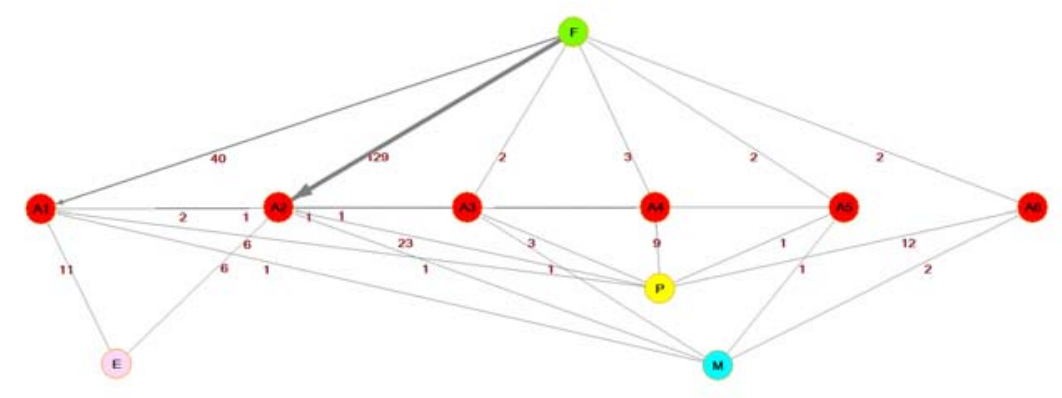

Figure 5 Cascading effects between causal factors and detailed accident types

In Figure 6(a) and (b), "P14 (illegally entering into the track area)", "F7 (communication and signal failure)" and "F2 (vehicle failure)" have the most number of links with accidents, which suggest that these casual factors are closely related to the occurrence of accident. Besides, poor management leads to "P2 (carrying dangerous articles)" appearing 8 times. That is to say, strengthening corresponding management can indirectly reduce accidents. It is noting that cascading effects in Figure 6(c) and (d) is not significant compared with person-type and facility-type factors. 


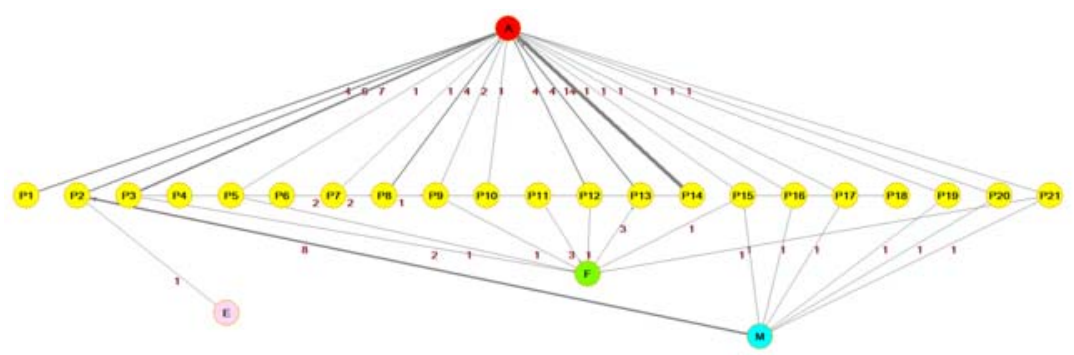

(a) detailed person-type factors

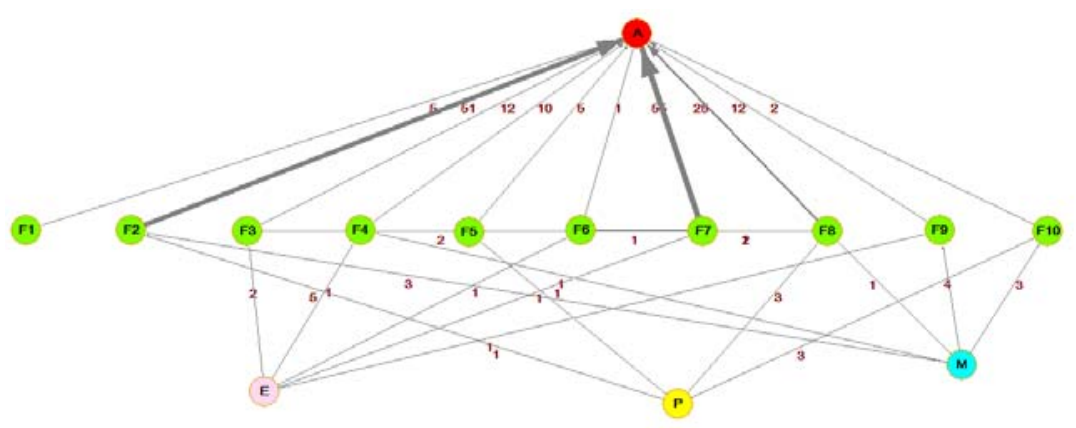

(b) detailed facility-type factors

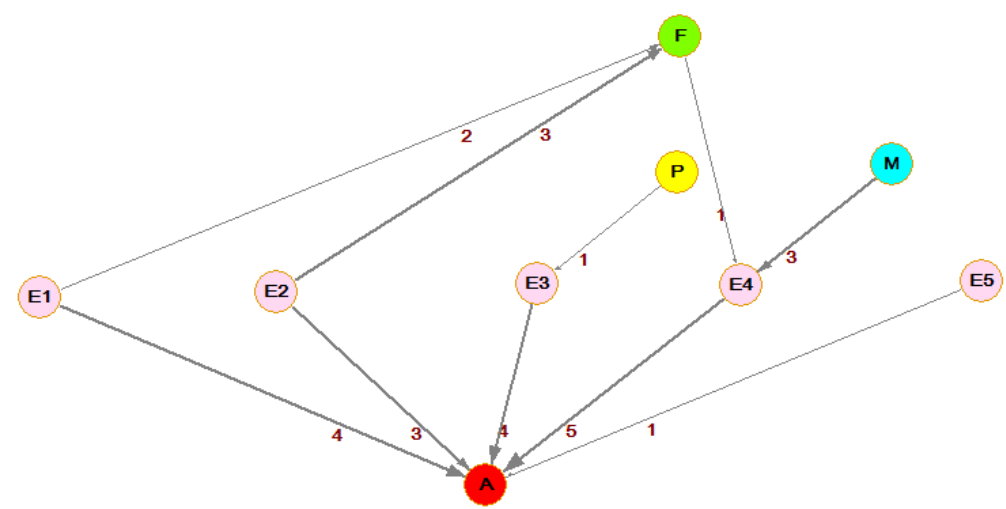

(c) detailed environment-type factors

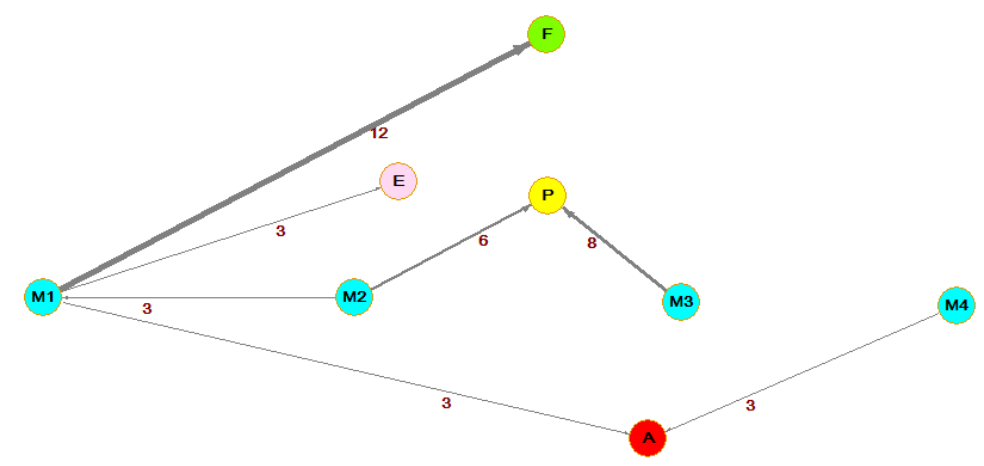

(d) detailed management-type factors

Figure 6 Cascading effects between detailed causal factors and other factors 


\subsection{Local View Analysis}

\subsubsection{Node Degree}

In directed networks, degree includes in-degree and out-degree. From the aspects of MOAN, the in-degree value of the node is the number of interdependencies from other causal factors, while the out-degree value of the node is the number of interdependencies to other factors. The calculation of degree is defined by Equations $(4) \sim(6)$.

$$
\begin{aligned}
& k_{i}^{\text {in }}=\sum_{j=1}^{N} a_{j i}, \\
& k_{i}^{\text {out }}=\sum_{j=1}^{N} a_{i j}, \\
& k_{i}=k_{i}^{\text {in }}+k_{i}^{\text {out }},
\end{aligned}
$$

where $k_{i}^{\text {in }}, k_{i}^{\text {out }}$ presents the in-degree and the out-degree of node $i$ respectively, $k_{i}$ presents the total degree.

The average degree of MOAN is 4.52, indicating that each causal factor connect on average with four or five other causal factors/accident types. From the view of out-degree, the maximum value does not exceed 9 , and the result is relatively uniform, which also makes the safety management more difficult.

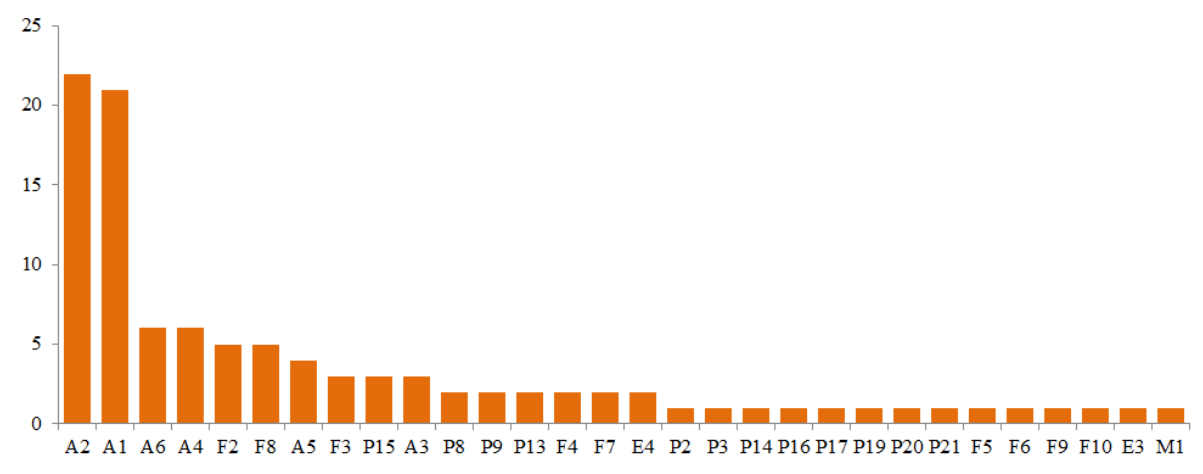

Figure 7 Values of in-degree

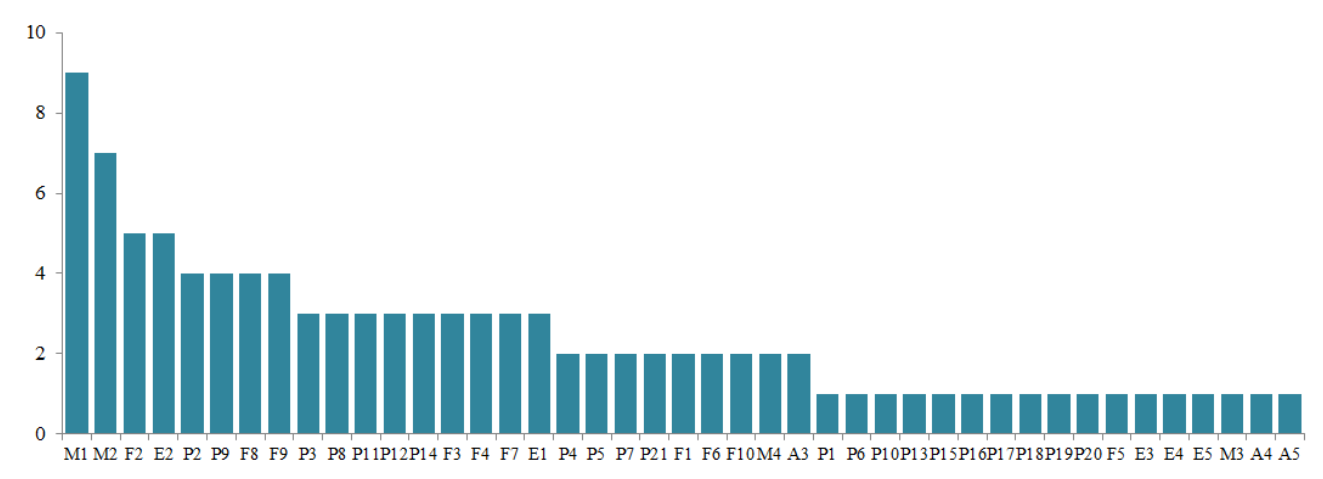

Figure 8 Values of out-degree 


\subsubsection{Node Strength}

Similarity with degree, in directed network, node strength includes in-strength and outstrength. Node strength can further reflect the weight of the interaction among nodes. Generally, a node with large strength value plays an important role in the network. The calculation of strength is defined by Equations $(7) \sim(9)$.

$$
\begin{aligned}
& S_{i}^{\text {in }}=\sum_{j=1}^{N} w_{j i}, \\
& S_{i}^{\text {out }}=\sum_{j=1}^{N} w_{i j}, \\
& S_{i}=S_{i}^{\text {in }}+S_{i}^{\text {out }},
\end{aligned}
$$

where $S_{i}^{\text {in }}, S_{i}^{\text {out }}$ presents the incoming weight and outgoing weight to the node $i$ respectively, $S_{i}$ presents the total strength.

As shown in Figure 9, "A2 (train delay)" and "A1 (train suspension)" are the two highest indegree factors. This indicates that they are the biggest risk recipients and many causal factors can result in the two accidents. "F7 (communication and signal failure)" and "F2 (vehicle failure)" have the highest out-degree. This indicates that they belongs to the primary causal factor, which is easy to cause other faults and is the great risk source. So they should be treated with high priority.

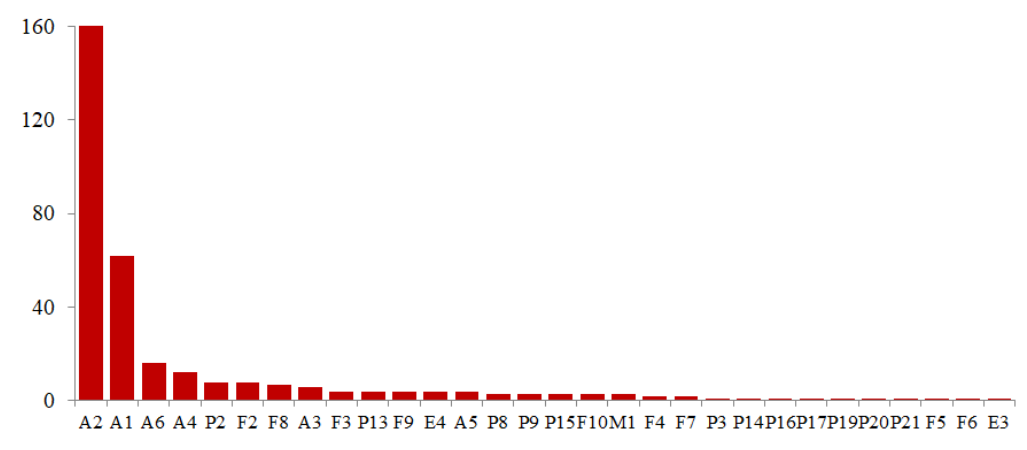

Figure 9 Values of in-strength

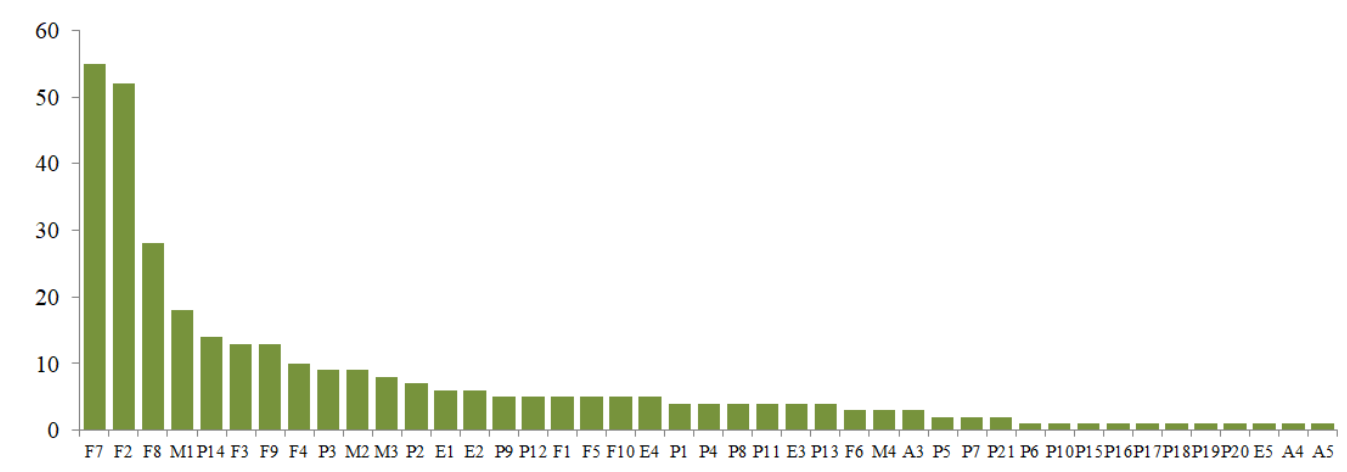

Figure 10 Values of out-strength 


\subsubsection{Betweenness Centrality}

Betweenness centrality is used to measure the extent to which a node can function as a point of control in the network. Obviously, nodes with high betweenness centrality play a significant intermediary role in the communication. It is defined by Equation (10).

$$
C_{b}(s)=\sum_{i \neq s \neq j} \frac{g_{s}(i, j)}{g(i, j)},
$$

where $g(i, j)$ is the total number of shortest paths connecting node $i$ and node $j, g_{s}(i, j)$ is the number of the shortest paths connecting node $i$ and node $j$ and through node $s$.

As shown in Figure 11, the betweenness centrality of nodes is generally small, and the maximum value is merely 0.011, which is related to the shortness of the event chain. Nodes with high betweenness centrality are "F2 (vehicle failure)", "F8 (power supply system failure)", "P9 (being pinched in train door)" and "M1 (poor routine safety maintenance and inspection)" in turn. What's more, the out-degree and in-degree values of these nodes are also relatively high, so they play a crucial role in risk spread. In addition, the sum of betweenness centrality of these four causal factors accounts for $61.5 \%$, indicating that more than a half of the critical path will pass through them, so effectively tackling these key causal factors can slow down the fault diffusion.

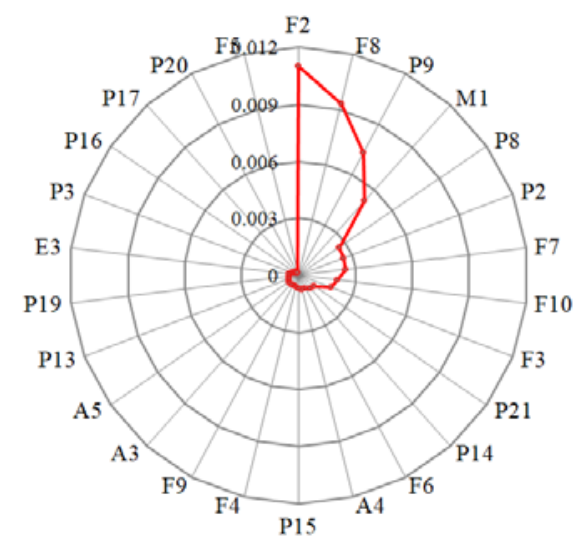

Figure 11 Values of betweenness centrality

\subsubsection{Cluster Coefficient}

Clustering coefficient describes the degree of cliquishness from a local perspective. Nodes with large clustering coefficient tend to be the central component of the network. It is defined by Equation (11).

$$
C_{i}=\frac{2 E_{i}}{k_{i}\left(k_{i}-1\right)}
$$

where $k_{i}$ is the number of neighboring nodes of node $i, E_{i}$ is the actual number of edges connected by node $i$ to its neighboring nodes, and $\frac{k_{i}\left(k_{i}-1\right)}{2}$ is the maximum number of possible edges connected by node $i$ to its neighboring nodes.

The average clustering coefficient of MOAN is 0.127 , implying the network has a high degree of cluster together. As shown in Figure 12, nodes with the highest clustering coefficient values 
are "P4 (piling into the train)", "P5 (opening the door forcedly)", and "E4 (smoke)". So it is quite necessary to control these causal factors with large clustering coefficient.

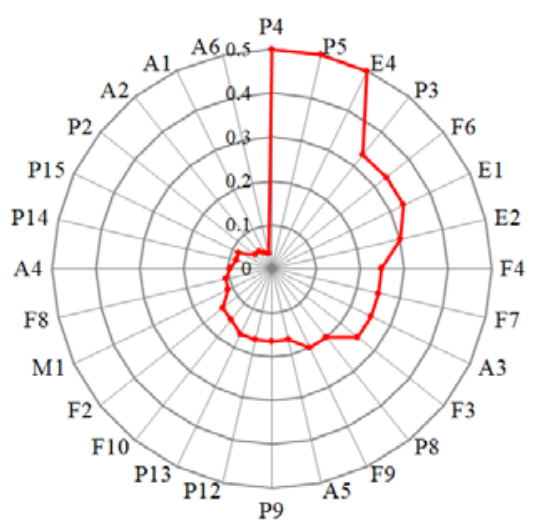

Figure 12 Values of cluster coefficient

\section{Conclusion and Future Work}

In this paper, structural properties from both global and local views are made using complex network theory. Research results show: 1) Facility-type factors are closely related to accidents in the least path length, and the number of accidents caused by facility-type factors is the highest. 2) "A2 (train delay)" and "A1 (train suspension)" rank in the top two among accident types. 3) "M1 (poor routine safety maintenance and inspection)", "M2 (inadequate education and training)", "F2 (vehicle failure)" and "E2 (severe weather)" have high out-degree; "F7 (communication and signal failure)", "F2 (vehicle failure)", and "F8 (power supply failure)" have high out-strength; "F2 (vehicle failure)", "F8 (power supply system failure)", "P9 (being pinched in train door)", and "M1 (poor routine safety maintenance and inspection)" have large betweenness centrality values; "P4 (piling into the train)", "P5 (opening the door forcedly)", and "E4 (smoke)" have large clustering coefficient values. These nine causal factors are the key causal factors from local views.

The paper aims to analyze the topological structure of directed weighted MOAN through complex network, and the results obtained from global and local views are of great reference value for improving metro operation safety. However, the following studies could be further discussed in the future. First, dynamic research mechanisms could be established to solve future problems with metro operation safety. Second, the metro operation accident database that presented in the paper can be further compared with foreign ones. Third, expert analysis mechanisms could be introduced to broaden the analysis perspective, so that the research is more in line with reality and can solve related problems more efficiency.

\section{Declaration of Competing Interest}

The authors declare that they have no known competing financial interests or personal relationships that could have appeared to influence the work reported in this paper. 


\section{References}

[1] Waterson P, Jenkins D P, Salmon P M, et al. 'Remixing rasmussen': The evolution of Accimaps within systemic accident analysis. Applied Ergonomics, 2017, 59: 483-503.

[2] Zhan Q J, Zheng W, Zhao B B. A hybrid human and organizational analysis method for railway accidents based on HFACS-Railway Accidents (HFACS-RAs). Safety Science, 2017, 91: 232-250.

[3] Goncalves Filho A P, Jun G T, Waterson P. Four studies, two methods, one accident - An examination of the reliability and validity of Accimap and STAMP for accident analysis. Safety Science, 2019, 113: 310-317.

[4] Lee S, Moh Y B, Tabibzadeh M, et al. Applying the AcciMap methodology to investigate the tragic Sewol Ferry accident in South Korea. Applied Ergonomics, 2017, 59: 517-525.

[5] Zhou J L, Lei Y. Paths between latent and active errors: Analysis of 407 railway accidents/incidents' causes in China. Safety Science, 2018, 110: 47-58.

[6] Ouyang M, Hong L, Yu M H, et al. STAMP-based analysis on the railway accident and accident spreading: Taking the China-Jiaoji railway accident for example. Safety Science, 2010, 48(5): 544-555.

[7] Wei Y C, Chen L, Qi Y, et al. A complex network method in criticality evaluation of air quality standards. Sustainability, 2019, 11(14): 3920.

[8] Zhao Z B, Chen D L, Wang L Q, et al. Credit risk diffusion in supply chain finance: A complex networks perspective. Sustainability, 2018, 10(12): 4608.

[9] He Q, Cao X S. Pattern and influencing factors of foreign direct investment networks between countries along the "Belt and Road" regions. Sustainability, 2019, 11(17): 4724.

[10] Dzafic I, Jabr R A, Hrnjic T. Hybrid state estimation in complex variables. IEEE Transactions on Power Systems, 2018, 33(5): 5288-5296.

[11] Fang Q X, Peng J G. Synchronization of fractional-order linear complex networks with directed coupling topology. Physica A, 2018, 490: 542-553.

[12] Zhou Z P, Irizarry J, Li Q M. Using network theory to explore the complexity of subway construction accident network (SCAN) for promoting safety management. Safety Science, 2014, 64: 127-136.

[13] Deng Y L, Song L L, Zhou Z P, et al. An approach for understanding and promoting coal mine safety by exploring coal mine risk network. Complexity, 2017, 2017: 1-17.

[14] Yang Y, Xu K J, Xiang H H. Analysis on Chinese airline network invulnerability. Journal of Systems Science and Information, 2019, 7(4): 359-372.

[15] Ma X, Li K P, Luo Z Y, et al. Analyzing the causation of a railway accident based on a complex network. Chinese Physics B, 2014, 23(2): 028904.

[16] Li Q M, Song L L, List G F, et al. A new approach to understand metro operation safety by exploring metro operation hazard network (MOHN). Safety Science, 2017, 93: 50-61.

[17] Liu J T, Schmid F, Wei Z, et al. Understanding railway operational accidents using network theory. Reliability Engineering \& System Safety, 2019, 189: 218-231.

[18] Lam C Y, Tai K. Network topological approach to modeling accident causations and characteristics: Analysis of railway incidents in Japan. Reliability Engineering \& System Safety, 2020, 193: 106626.

[19] Zhou J, Xu W X, Guo X, et al. A method for modeling and analysis of directed weighted accident causation network (DWACN). Physica A, 2015, 437: 263-277.

[20] Hou G Y, Jin C, Xu Z D, et al. Exploring evolutionary features of directed weighted hazard network in the subway construction. Chinese Physics B, 2019, 28(3): 038901.

[21] Du Z Y, Tang J J, Qi Y, et al. Identifying critical nodes in metro network considering topological potential: A case study in Shenzhen city, China. Physica A, 2020, 539: 22926. 